\title{
Phase 1 Studies of Poziotinib, an Irreversible Pan-HER Tyrosine Kinase Inhibitor in Patients with Advanced Solid Tumors
}

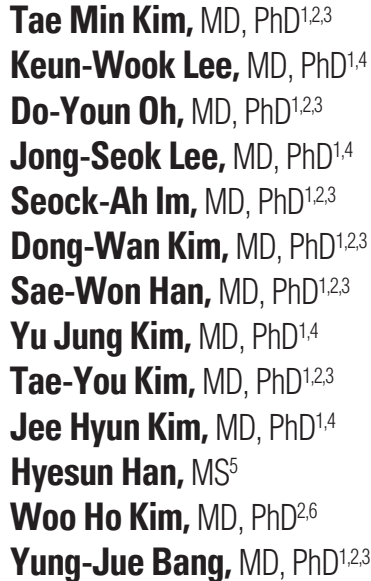

${ }^{1}$ Department of Internal Medicine, Seoul National University College of Medicine, Seoul, ${ }^{2}$ Seoul National University Cancer Research Institute, Seoul, ${ }^{3}$ Department of Internal Medicine, Seoul National University Hospital, Seoul, ${ }^{4}$ Department of Internal Medicine, Seoul National University Bundang Hospital, Seongnam, ${ }^{5}$ Hanmi Pharm. Co., Ltd., Seoul, ${ }^{6}$ Department of Pathology, Seoul National University College of Medicine, Seoul, Korea

Correspondence: Yung-Jue Bang, MD, PhD Department of Internal Medicine, Seoul National University College of Medicine, 101 Daehak-ro, Jongno-gu, Seoul 03080, Korea

Tel: 82-2-2072-2390

Fax: 82-2-764-2199

E-mail: bangyj@snu.ac.kr

Received June 22, 2017

Accepted August 18, 2017

Published Online August 29, 2017

*Data from these studies were presented in part at the annual meetings of the American Society of Clinical Oncology 2012 and 2013, Chicago, IL, USA; and 15th World Conference on

Lung Cancer 2013, Sydney, Australia.

\begin{abstract}
Purpose
Poziotinib, a pan-human epidermal growth factor receptor 2 (HER) tyrosine kinase inhibitor, has shown potent activity against wild type of epidermal growth factor receptor (EGFR) family kinases including EGFR, HER2, and HER4 and EGFR-mutant cells in vitro. Two phase I studies were conducted to determine the maximum tolerated dose (MTD), pharmacokinetics, safety, and antitumor activity against advanced solid tumors.
\end{abstract}

\section{Materials and Methods}

Standard 3+3 dose escalation scheme using two different dosing schedules were studied: once daily, 14-day on, and 7-day off (intermittent schedule); and once daily continuous dosing with food effect. Additional patients were enrolled in an expansion cohort.

\section{Results}

A total of 75 patients were enrolled in the two studies. The most common drug-related treatment-emergent adverse events were diarrhea, rash, stomatitis, pruritus, and anorexia. Doselimiting toxicities were grade 3 diarrhea in the intermittent schedule and grade 3 anorexia and diarrhea in the continuous dosing schedule. The MTDs were determined as $24 \mathrm{mg} /$ day in the intermittent dosing schedule and $18 \mathrm{mg} /$ day in the continuous dosing schedule. Eight (16\%) and $24(47 \%)$ of 51 evaluable patients in the intermittent schedule achieved partial response (PR) and stable disease (SD), respectively. Four (21\%) and six (32\%) of 19 evaluable patients in continuous dosing schedule achieved PR and SD, respectively. Patients with PR $(n=7)$ or $S D \geq 12$ weeks $(n=7)$ had HER2 amplification ( $n=7$; breast cancer, 5 ; and stomach cancer, 2) and EGFR amplification ( $\mathrm{n}=1$, squamous cell lung cancer).

\section{Conclusion}

Poziotinib was safe and well tolerated in patients with advanced solid tumors. It showed an encouraging activity against EGFR-mutant and HER2-amplified cancers.

\author{
Key words \\ EGFR mutation, HER2 amplification, Poziotinib, \\ Non-small-cell lung carcinoma
}




\section{Introduction}

The ErbB family of receptors is mutated or overexpressed in many solid tumors and aberrantly activated ErbB receptor signals have been associated with poor prognosis [1]. Among ErbB receptors, epidermal growth factor receptor (EGFR) and human epidermal growth factor receptor 2 (HER2) have been implicated as important therapeutic targets for lung, breast, and gastric cancers. The first-generation EGFR tyrosine kinase inhibitors (TKIs) including gefitinib and erlotinib reversibly inhibit EGFR tyrosine kinase by competing with adenosine triphosphate [1]. These have shown clinical benefits for EGFR-mutant non-small cell lung cancer (NSCLC) with median progression-free survivals of 9 to 11 months; however, acquired resistance to these TKIs is inevitable [2]. Due to limited clinical success of first-generation EGFR TKI, second-generation and irreversible EGFR TKIs have been developed for targeting EGFR, HER2, and ErbB4 such as dacomitinib and afatinib [3]. These second-generation EGFR TKIs have shown favorable survival outcomes in lung adenocarcinoma with activating EGFR mutations [4-6].

Poziotinib (HM781-36B) is an irreversible pan-HER TKI, which targets EGFR, HER2, and ErbB4. Poziotinib shows good in vitro activities in EGFR or HER2 expressing various cancer cell lines including EGFR TKI-resistant lung cancer cells. Poziotinib effectively inhibited ErbB family kinases $\left(\mathrm{IC}_{50}\right.$, $3.2,2.2,5.3$, and $23.5 \mathrm{nM}$ against $E G F R^{\text {wild-type }}, E G F R^{\mathrm{T} 790 \mathrm{M} / \mathrm{L} 858 \mathrm{R}}$, HER2, and HER4, respectively) [7]. In addition, HER2amplified gastric cancer cells and breast cancer cells were sensitive to poziotinib alone ( $\mathrm{IC}_{50}, 1-4 \mathrm{nM}$ and $1-9 \mathrm{nM}$, respectively) or poziotinib in combination with various chemotherapeutic agents $[8,9]$.

Here, we conducted the first-in-human phase I studies of poziotinib to determine the maximum tolerated dose (MTD), pharmacokinetics, safety, and antitumor activity in patients with advanced solid tumors.

\section{Materials and Methods}

\section{Patients}

Eligible patients were $\geq 19$ years with histologically or cytologically confirmed advanced solid tumor, Eastern Cooperative Oncology Group performance status $\leq$; with previous chemotherapy, a life expectancy greater than 12 weeks; and adequate bone marrow (absolute neutrophil count $\geq 1,500 / \mathrm{mm}^{3}$, platelet $\geq 100,000 / \mathrm{mm}^{3}$, and hemoglobin $\geq 9.0 \mathrm{~g} / \mathrm{dL}$ ), renal (serum creatinine $\leq 1.5 \mathrm{mg} / \mathrm{dL}$ ) and hepatic (serum transaminases $\leq 3 \times$ upper limit of normal and total bilirubin $\leq 2 \mathrm{mg} / \mathrm{dL}$ ) functions. Patients who had undergone radiation therapy or surgery within 4 weeks prior to study were excluded. Patients were excluded if they had uncontrolled infection, ileus, metastasis to central nervous system, class III or IV heart failure or left ventricular ejection fraction $<40 \%$, gastrointestinal malabsorption or difficulty in taking oral medication, or taken other investigational products within 30 days before screening.

\section{Study design and treatment}

We conducted two phase I, open label studies to determine the MTD for two different dosing schedules. In NCT0145571 (study A), poziotinib tablet was administered with water once daily on an intermittent basis (14-day on and 7-day off) schedule at multiple dose levels $(0.5,1,2,4,8,12,16,20,24$, and $32 \mathrm{mg}$ ) (n=55, study A), and in NCT01455584 (study B), poziotinib was given once daily in a continuous dosing fashion, at dose levels of 12, 18, and $24 \mathrm{mg}$ and $16 \mathrm{mg}$ with food in the fasting or fed state by period in a cross-over design $(n=20$, study B). In the dose escalation part for both studies, cohorts of three to six patients were treated at increasing doses of poziotinib to determine the MTD. A traditional 3+3 dose-escalation design was used with MTD defined as the dose where dose-limiting toxicities (DLTs) observed in one or more out of six patients in the first cycle. Once the MTD was identified, 12 patients were enrolled in the expansion part (study A, only). Food effect was conducted based on a $2 \times 2$ cross-over design (fasting-fed sequence versus fed-fasting sequence after randomization) once the recommended dose $(\mathrm{RD})$ was determined (study B, only).

\section{Pharmacokinetic analyses}

In study A, serial blood and urine samples were collected on day 1 for up to 24 hours (just before administration, 0.5, $1,1.5,2,3,4,6,8,10,12$, and 24 hours after dosing) and day 14 of cycle 1 up to 48 hours (just before administration, 0.5 , $1,1.5,2,3,4,6,8,10,12,24$, and 48 hours after dosing) $(\mathrm{n}=52)$. To assess the food effect on pharmacokinetic (PK) of poziotinib in study B, eigh additional patients were enrolled, and blood samples were taken on day 1 and day 8 up to 24 hours (just before administration, 0.5, 1, 1.5, 2, 3, 4, 6, 8, 12, and 24 hours after dosing) on fasting or fed condition $(n=8)$. Plasma samples were analyzed for poziotinib concentration at BioInfra Co., Ltd. (Suwon, Korea). The analytes were extracted from heparinized human plasma. The analytes were then separated and detected by validated ultra-performance liquid chromatography with tandem mass spectrometry [10]. Noncompartmental analysis for plasma concentration was performed using Phoenix WinNonlin Software ver. 6.3 
(Pharsight, Mountain View, CA).

\section{Efficacy and safety analyses}

Adverse events (AEs) and treatment-emergent AEs (TEAEs) were evaluated using National Cancer Institute Common Terminology Criteria for Adverse Events ver. 3.0. DLTs were defined as grade $\geq 3$ non-hematologic toxicities (except for alopecia), grade $\geq 3$ diarrhea, nausea and vomiting despite maximal anti-diarrheal, anti-emetics medication, grade 4 neutropenia sustained for 7 days or more or febrile neutropenia, grade $\geq 3$ neutropenic infection accompanying grade 4 neutropenia or grade 4 thrombocytopenia. Treatment response to poziotinib was evaluated every two cycles based on the Response Evaluation Criteria in Solid Tumors criteria (RECIST) ver. 1.0 [11]. Progression-free survival (PFS) was measured as the interval between poziotinib first dose administration and the date when disease progression by RECIST ver. 1.0 or death was first documented.

\section{Biomarker and molecular analyses}

Plasma or tumor samples in the expansion cohort $(n=12$, study A) and in the continuous and food effect cohort ( $\mathrm{n}=20$, study B) were collected for EGFR mutation on exons 18-21,
KRAS mutation on exon 2, BRAF mutation on exon 15, and PIK3CA mutation on exons 9 and 20 using a peptide nucleic acid (PNA)-mediated clamping polymerase chain reaction (PCR) method (PANAGENE, Inc., Daejeon, Korea) [12]. Plasma hepatocyte growth factor (HGF) concentration was determined using quantitative sandwich enzyme immunoassay technique (Seoul Clinical Laboratories Co., Ltd., Yongin, Korea). Plasma and tumor samples derived from patients $(\mathrm{n}=14$, studies A and B) with partial response (PR) or stable disease (SD) $\geq 12$ weeks were examined for EGFR (EGFR/ CEP7 probe, Abbott Laboratories, Des Plaines, IL), HER2 (HER2/CEP7 probe, Abbott Laboratories), HER3 (HER3/ CEN12 probe, ZytoVision GmbH, Bremerhaven, Germany), or HER4 (HER4/2q11 probe, ZytoVision GmbH) amplification and EGFR or HER2 mutation (PNA-mediated clamping $\mathrm{PCR}$ or direct sequencing).

\section{Ethical statement}

These studies were conducted in compliance with the Declaration of Helsinki and following the International Conference on Harmonization Good Clinical Practice Guidelines. In addition, these studies were approved by the Institutional Review Board of each participating center. Written informed consents were obtained.

Table 1. Patient characteristics

\begin{tabular}{|c|c|c|}
\hline Characteristic & $\begin{array}{l}\text { Intermittent dosing schedule } \\
\qquad(\mathrm{n}=55)\end{array}$ & $\begin{array}{l}\text { Continuous dosing schedule } \\
\qquad(\mathrm{n}=20)\end{array}$ \\
\hline \multicolumn{3}{|l|}{ Sex } \\
\hline Male & $31(56)$ & $13(65)$ \\
\hline Female & $24(44)$ & 7 (35) \\
\hline Age (yr) & $55(25-83)$ & $55(32-77)$ \\
\hline \multicolumn{3}{|l|}{ ECOG PS } \\
\hline 0 & $30(55)$ & $8(40)$ \\
\hline 1 & $22(40)$ & $12(60)$ \\
\hline 2 & $2(4)$ & 0 \\
\hline 3 & $1(2)$ & 0 \\
\hline \multicolumn{3}{|l|}{ Cancer } \\
\hline NSCLC & $22(40)$ & $5(25)$ \\
\hline Stomach & $10(18)$ & $3(15)$ \\
\hline Breast & $8(15)$ & $2(10)$ \\
\hline Colorectal & $9(16)$ & $6(30)$ \\
\hline Others & $6(11)$ & $4(20)$ \\
\hline \multicolumn{3}{|l|}{ Prior regimen } \\
\hline $1-2$ & $9(16)$ & $3(15)$ \\
\hline 3 & $9(16)$ & $5(25)$ \\
\hline$\geq 4$ & $37(67)$ & $12(60)$ \\
\hline
\end{tabular}

Values are presented as number (\%) or median (range). ECOG PS, Eastern Cooperative Oncology Group performance status; NSCLC, non-small cell lung cancer. 
Table 2. Poziotinib-related TEAEs $(\geq 10 \%)$

\begin{tabular}{|c|c|c|c|c|}
\hline \multirow{2}{*}{ TEAEs, preferred terms } & \multicolumn{2}{|c|}{ Intermittent dosing schedule $(\mathrm{n}=55)$} & \multicolumn{2}{|c|}{ Continuous dosing schedule $(n=20)$} \\
\hline & Grade 1-4 & $\geq$ Grade 3 & Grade 1-4 & $\geq$ Grade 3 \\
\hline Diarrhea & $48(87)$ & $8(15)$ & $20(100)$ & $3(15)$ \\
\hline Rash & $46(84)$ & $1(2)$ & $13(65)$ & - \\
\hline Stomatitis & $40(73)$ & - & $16(80)$ & $1(5)$ \\
\hline Pruritus & $33(60)$ & - & $11(55)$ & $1(5)$ \\
\hline Decreased appetite & $20(36)$ & $1(2)$ & $11(55)$ & $2(10)$ \\
\hline Acne & $17(31)$ & - & - & - \\
\hline Dermatitis acneiform & $2(4)$ & - & $6(30)$ & $2(10)$ \\
\hline Palmar-plantar erythrodysaesthesia syndrome & $18(33)$ & - & $3(15)$ & - \\
\hline Rhinorrhea & $14(26)$ & - & $6(30)$ & - \\
\hline Paronychia & $13(24)$ & - & $13(65)$ & - \\
\hline Mucosal inflammation & $13(24)$ & $1(2)$ & $6(30)$ & - \\
\hline Nausea & $11(20)$ & $1(2)$ & $4(20)$ & - \\
\hline Vomiting & $10(18)$ & $1(2)$ & $4(20)$ & - \\
\hline Fatigue & $8(15)$ & - & $3(15)$ & - \\
\hline Skin exfoliation & $8(15)$ & - & - & - \\
\hline Dry skin & $6(11)$ & - & $6(30)$ & $2(10)$ \\
\hline Weight decreased & $6(11)$ & - & - & - \\
\hline
\end{tabular}

Values are presented as number (\%). TEAEs, treatment-emergent adverse events.

Table 3. DLTs in dose escalation and extension phases

\begin{tabular}{|c|c|c|c|c|c|c|}
\hline \multirow{2}{*}{ Dose (mg) } & \multicolumn{3}{|c|}{ Intermittent dosing schedule } & \multicolumn{3}{|c|}{ Continuous dosing schedule } \\
\hline & No. of patients & No. of DLTs & DLT details & No. of patients & No. of DLTs & DLT details \\
\hline 0.5 & 3 & - & - & - & - & - \\
\hline 1 & 3 & - & - & - & - & - \\
\hline 2 & 3 & - & - & - & - & - \\
\hline 4 & 3 & - & - & - & - & - \\
\hline 8 & 3 & - & - & - & - & - \\
\hline 12 & 6 & 1 & Diarrhea & 3 & - & - \\
\hline 16 & 6 & 1 & Diarrhea & - & - & - \\
\hline 18 & - & - & - & 6 & 1 & Decreased appetite \\
\hline 20 & 3 & - & - & - & - & - \\
\hline 24 & 6 & 1 & Diarrhea & 3 & 1 & $\begin{array}{c}\text { Diarrhea } \\
\text { Drug compliance }<80 \%\end{array}$ \\
\hline 32 & 7 & 2 & Diarrhea & - & - & - \\
\hline 24 Extension & 12 & 5 & $\begin{array}{l}3 \text { Diarrhea, } \\
1 \text { nausea, } \\
\text { cosal inflammation }\end{array}$ & - & - & - \\
\hline
\end{tabular}

DLT, dose-limiting toxicity. 


\section{Results}

\section{Patients}

A total of 75 patients were enrolled in both studies (55 in the intermittent schedule and 20 patients in the continuous dosing schedule, respectively). Study A included 43 patients in the dose escalation portion and 12 patients in the expansion cohort at the MTD. Study B included 12 patients in the dose escalation portion and eight patients in the food effect cohort. Nearly one-third of the patients $(\mathrm{n}=27)$ in both studies had a diagnosis of NSCLC and the majority of patients $(65 \%)$ received fourth-line or above chemotherapeutic regimens (Table 1).

\section{Safety profiles}

All 75 patients were included in the safety analysis. Fiftytwo patients $(95 \%)$ in the intermittent schedule and 20 patients $(100 \%)$ in the continuous schedule experienced at least one drug-related AE. The most common drug-related TEAEs were diarrhea, rash, stomatitis, pruritus, and anorexia (Table 2). DLTs in the intermittent dosing schedule were grade 3 diarrhea in five patients (one each at 12, 16, $24 \mathrm{mg}$ levels, and two at $32 \mathrm{mg}$ ) in dose escalation part and grade 3 diarrhea in three patients, grade 3 nausea in one patient, and grade 3 mucosal inflammation in one patient in $24 \mathrm{mg}$ expansion part (Table 3). DLTs in the continuous dosing schedule were grade 3 anorexia (one at $18 \mathrm{mg}$ ), grade 3 diarrhea with anorexia (one at $24 \mathrm{mg}$ ), and drug compliance as defined by actual dosage administered/dosage that should be administered $\times 100<80 \%$ due to grade 2 toxicities such as anorexia, rash, nausea, fatigue and diarrhea (one at $24 \mathrm{mg}$ ) (Table 3). The MTDs were determined as $24 \mathrm{mg} /$ day in the intermittent dosing schedule and $18 \mathrm{mg} /$ day in the continuous dosing schedule.

\section{Efficacy}

There were 51 evaluable patients by RECIST ver. 1.0 in the intermittent dosing schedule (study A) and out of those, eight patients $(16 \%$ ) achieved PR (breast cancer, $n=4$; NSCLC, $\mathrm{n}=2$; and gastric and colorectal cancers, $\mathrm{n}=1$, respectively) and $24(47 \%)$ had SD (Fig. 1A). The disease control rate (DCR, $\mathrm{PR}+\mathrm{SD}$ ) was $63 \%$. The median PFS was 12.0 weeks (95\% confidence interval [CI], 7.4 to 16.5$)$ for all patients and 13.4 weeks (95\% CI, 11.5 to 18.9) in patients treated with at least $8 \mathrm{mg} /$ day $(\mathrm{n}=39)$. All of the three patients $(100 \%)$ receiving $8 \mathrm{mg} /$ day developed a rash, a well-known AE related with inhibition of wild-type EGFR. Considering Ki-67-positive cells decreased after treatment with $\geq 8 \mathrm{mg}$ poziotinib, the minimum effective dose for poziotinib was estimated to be $8 \mathrm{mg} /$ day.

There were 19 evaluable patients in the continuous dosing schedule (study B). Four patients $(21 \%)$ including two breast and one common bile duct cancers and one NSCLC achieved PR and six (32\%) achieved SD; the DCR was 53\% (Fig. 1B). The median PFS was 9.0 weeks (95\% CI, 5.7 to 39.7). Among 27 NSCLC patients, 26 had received prior EGFR TKIs (firstgeneration EGFR TKIs, $\mathrm{n}=26$; and second-generation EGFR TKIs, $\mathrm{n}=3)$. Three $(11 \%)$ and $11(41 \%)$ patients achieved PR and SD, respectively (Fig. 1C). The median PFS was 11.4 weeks (range, 5.6 to 17.4 weeks).

\section{Pharmacokinetics}

PK data for poziotinib was previously reported [10]. Briefly, dose-proportional pharmacokinetics profiles were observed in terms of $\mathrm{C}_{\max }$ and area under plasma concentration-time curve (AUC) in dose range of 0.5 to $24 \mathrm{mg}$ on day 1. Absorption rate of poziotinib was moderate to high with a median $\mathrm{T}_{\max }$ between 0.6 and 4 hours and mean terminal half-lives ranged from 5.09 to 9.92 hours in day 1. PK parameters were similar between single (on day 1 ) and multiple oral doses (on day 14).

There was minimal difference in the geometric mean AUC values of poziotinib between dosing under fed conditions and dosing under fasting conditions. The geometric mean $\mathrm{C}_{\max }$ of poziotinib decreased approximately by $27 \%$ under fed conditions compared with fasting conditions. The median $\mathrm{T}_{\max }$ of poziotinib was delayed from $1.26(0.95,3.00)$ to $3.01(1.00,7.85)$ hours with food intake (S1 Table).

\section{Molecular studies}

Tissue $(\mathrm{n}=9)$ and plasma $(\mathrm{n}=12)$ samples in the expansion part of study A (intermittent dosing schedule) and plasma $(n=9)$ samples in the continuous dosing schedule (study B) were collected for genetic studies. EGFR exon 19 deletion was observed in five NSCLC patients' tissue samples, two of which harbored concomitant $E G F R^{\mathrm{T} 790 \mathrm{M}}$ mutation. Plasma or tumor samples showed EGFR exon 19 deletions $(\mathrm{n}=6)$,

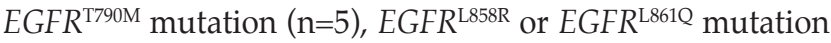
$(\mathrm{n}=2)$, and KRAS mutation $(\mathrm{n}=1)$. Molecular profiles, HGF concentrations and best response are described in S2 Table. Patients with PR $(n=7)$ or $\mathrm{SD} \geq 12$ weeks $(\mathrm{n}=7)$ had HER2 amplification ( $\mathrm{n}=7$; breast cancer, 5 ; and stomach cancer, 2$)$ and EGFR amplification ( $\mathrm{n}=1$, squamous cell lung cancer) (S3 Table). 


\section{A}

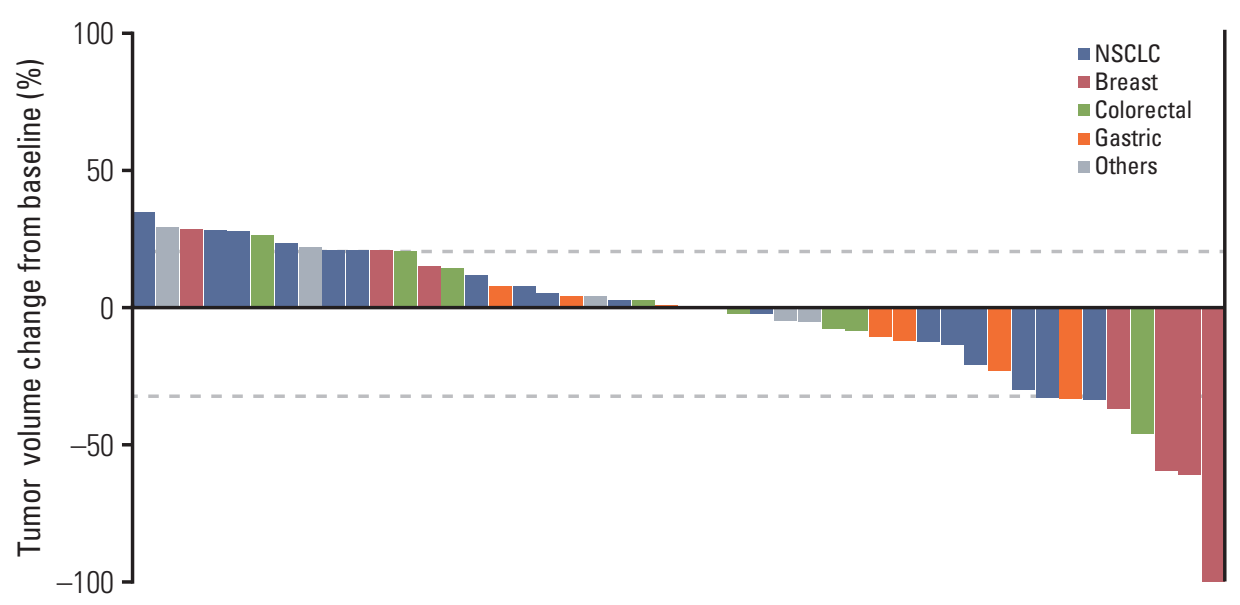

B

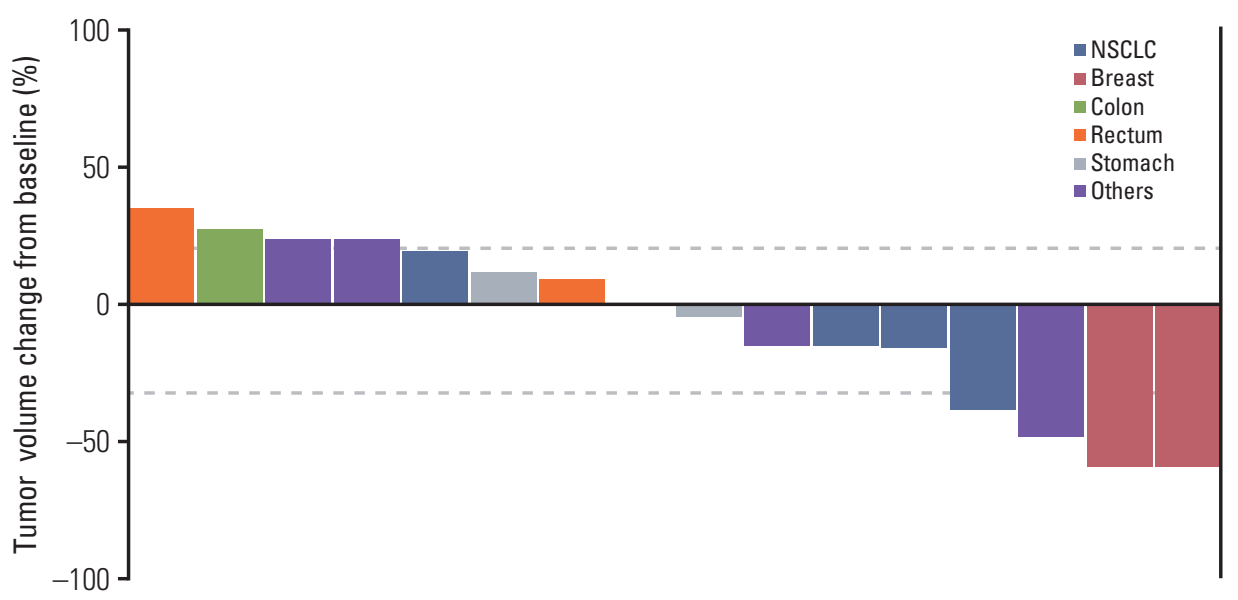

C

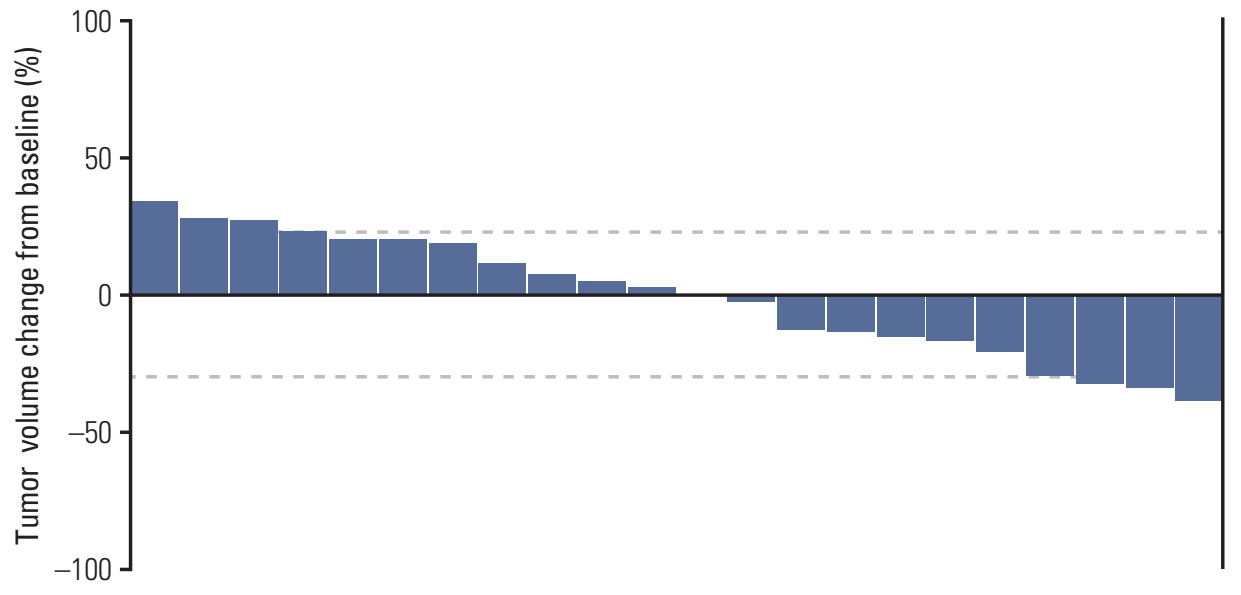

Fig. 1. (A) Waterfall plot shows tumor size changes of target lesions in patients who received poziotinib once daily on a 14-day on and 7-day off schedule $(n=46)$. (B) Waterfall plot shows tumor size changes of target lesions in patients treated with poziotinib once daily continuously $(\mathrm{n}=16)$. (C) Waterfall plot for non-small cell lung cancer (NSCLC) patients. 


\section{Discussion}

Poziotinib is an irreversible pan-HER TKI that has shown a manageable safety profile in our first-in-human studies, very similar to those seen with other TKIs. The MTDs were determined as $24 \mathrm{mg} /$ day in the intermittent dosing schedule 2 (study A) and $18 \mathrm{mg} /$ day in study B (continuous dosing) with a RD of $16 \mathrm{mg}$ once daily. A linear increase in poziotinib exposure with increasing doses was observed in PK analyses. The best overall responses were $15.7 \%$ in study $A$ and $21.1 \%$ in study B, respectively. In addition, encouraging efficacy was observed in EGFR-mutant NSCLC and HER2-amplified breast or stomach cancers. The most common AEs observed were diarrhea, skin rash, stomatitis, and pruritus and DLT was diarrhea, in line with other irreversible pan-HER TKIs $[13,14]$.

To evaluate the PK profile, PK and plasma assessments were performed on days 1 and 14 of the cycle 1 from 52 of a total of 55 patients in study A. The exposure to poziotinib increased generally dose-proportionally at doses up to 24 $\mathrm{mg} /$ day (i.e., MTD) and these data indicate that human PK of poziotinib showed dose dependency on oral absorption. In addition, the PK parameters relevant to poziotinib exposure at steady state (on day 14) were similar to those obtained following a single dose (on day 1) suggesting no accumulation of poziotinib after multiple administrations. In terms of food effect, $\mathrm{PK}$ data showed delay in $\mathrm{T}_{\max }$ and decreased in $\mathrm{C}_{\max }$ of poziotinib with food, but there were no significant differences in the exposure (AUC). Therefore, the anticancer activity of poziotinib is not altered by food ingestion.

In the present studies, EGFR, HER2, KRAS, BRAF, PIK3CA mutations, EGFR and HER2 amplifications, as well as plasma HGF concentrations were analyzed in an exploratory manner. In terms of the best overall response by genetic status, one NSCLC patient with EGFR exon 19 deletion mutation achieved SD and two NSCLC patients with EGFR exon 19 deletion and T790M mutation showed SD and PR, respectively. Although two NSCLC patients progressed despite the presence of EGFR activating mutations, this might be due to unknownresistance mechanisms associated with EGFR TKIs, considering that the tumor sample was collected after previous treatment with EGFR TKIs. Taken together, after treatment with poziotinib, SD or PR was confirmed in three of six NSCLC patients with EGFR exon 19 deletion mutation or concomitant EGFR exon 19 deletion and T790M mutation. Poziotinib showed encouraging efficacy in EGFR-mutant NSCLC after failure of prior EGFR TKIs (PR, 12.5\%) unlike other pan-HER inhibitors [13,14]. In addition, half of patients with clinical benefit (PR or $\mathrm{SD} \geq 12$ weeks) had HER2 amplification, mostly observed in patients with breast or stomach cancers. These results have led to the initiation of other poziotinib trials in various solid tumors, including EGFR-mutant lung adenocarcinoma as a first-line (NCT01819428) and as a second-line after failure to first-generation EGFR TKIs (NCT01718847), as well as in HER2-overexpressed breast and gastric cancers (NCT02418689, NCT02659514 and NCT01746771, respectively).

In conclusion, poziotinib is a promising irreversible panHER inhibitor against EGFR-mutant NSCLC as well as HER2-overexpressing breast and gastric cancers and was generally tolerated in heavily pre-treated cancer patients.

\section{Electronic Supplementary Material}

Supplementary materials are available at Cancer Research and Treatment website (http:// www.e-crt.org).

\section{Conflicts of Interest}

Conflict of interest relevant to this article was not reported.

\section{References}

1. Hynes NE, Lane HA. ERBB receptors and cancer: the complexity of targeted inhibitors. Nat Rev Cancer. 2005;5:341-54.

2. Chong CR, Janne PA. The quest to overcome resistance to EGFR-targeted therapies in cancer. Nat Med. 2013;19:1389-400.

3. Dienstmann R, De Dosso S, Felip E, Tabernero J. Drug development to overcome resistance to EGFR inhibitors in lung and colorectal cancer. Mol Oncol. 2012;6:15-26.

4. Sequist LV, Yang JC, Yamamoto N, O'Byrne K, Hirsh V, Mok $\mathrm{T}$, et al. Phase III study of afatinib or cisplatin plus pemetrexed in patients with metastatic lung adenocarcinoma with EGFR mutations. J Clin Oncol. 2013;31:3327-34.

5. Wu YL, Zhou C, Hu CP, Feng J, Lu S, Huang Y, et al. Afatinib versus cisplatin plus gemcitabine for first-line treatment of Asian patients with advanced non-small-cell lung cancer harbouring EGFR mutations (LUX-Lung 6): an open-label, randomised phase 3 trial. Lancet Oncol. 2014;15:213-22.

6. Janne PA, Ou SH, Kim DW, Oxnard GR, Martins R, Kris MG, et al. Dacomitinib as first-line treatment in patients with clinically or molecularly selected advanced non-small-cell lung cancer: a multicentre, open-label, phase 2 trial. Lancet Oncol. 
2014;15:1433-41.

7. Cha MY, Lee KO, Kim M, Song JY, Lee KH, Park J, et al. Antitumor activity of HM781-36B, a highly effective pan-HER inhibitor in erlotinib-resistant NSCLC and other EGFR-dependent cancer models. Int J Cancer. 2012;130:2445-54.

8. Nam HJ, Kim HP, Yoon YK, Hur HS, Song SH, Kim MS, et al. Antitumor activity of HM781-36B, an irreversible Pan-HER inhibitor, alone or in combination with cytotoxic chemotherapeutic agents in gastric cancer. Cancer Lett. 2011;302:155-65.

9. Kim HJ, Kim HP, Yoon YK, Kim MS, Lee GS, Han SW, et al. Antitumor activity of HM781-36B, a pan-HER tyrosine kinase inhibitor, in HER2-amplified breast cancer cells. Anticancer Drugs. 2012;23:288-97.

10. Noh YH, Lim HS, Jung JA, Song TH, Bae KS. Population pharmacokinetics of HM781-36 (poziotinib), pan-human EGF receptor (HER) inhibitor, and its two metabolites in patients with advanced solid malignancies. Cancer Chemother Pharmacol. 2015;75:97-109.

11. Therasse P, Arbuck SG, Eisenhauer EA, Wanders J, Kaplan RS, Rubinstein L, et al. New guidelines to evaluate the response to treatment in solid tumors. European Organization for Research and Treatment of Cancer, National Cancer Institute of the United States, National Cancer Institute of Canada. J Natl Cancer Inst. 2000;92:205-16.

12. Kim HJ, Lee KY, Kim YC, Kim KS, Lee SY, Jang TW, et al. Detection and comparison of peptide nucleic acid-mediated real-time polymerase chain reaction clamping and direct gene sequencing for epidermal growth factor receptor mutations in patients with non-small cell lung cancer. Lung Cancer. 2012;75:321-5.

13. Eskens FA, Mom CH, Planting AS, Gietema JA, Amelsberg A, Huisman $\mathrm{H}$, et al. A phase I dose escalation study of BIBW 2992, an irreversible dual inhibitor of epidermal growth factor receptor 1 (EGFR) and 2 (HER2) tyrosine kinase in a 2-week on, 2-week off schedule in patients with advanced solid tumours. Br J Cancer. 2008;98:80-5.

14. Janne PA, Boss DS, Camidge DR, Britten CD, Engelman JA, Garon EB, et al. Phase I dose-escalation study of the pan-HER inhibitor, PF299804, in patients with advanced malignant solid tumors. Clin Cancer Res. 2011;17:1131-9. 